\title{
Use of Radium223 in metastatic prostate cancer patient in treatment with denosumab: a potential trigger for Osteonecrosis of the jaw? A case report.
}

\author{
Francesco Pavese, Silvia Rotondaro \\ 1 University of Aquila \\ Funding: The author(s) received no specific funding for this work. \\ Potential competing interests: The author(s) declared that no potential competing interests exist.
}

\section{Abstract}

Radium-223 dichloride (Ra223) is an alpha-emitting radiopharmaceutical agent approved for the treatment of patients with castration-resistant prostate cancer (CRPC) with symptomatic bone metastases. Denosumab, a human monoclonal antibody targeting the key bone resorption mediator RANKL, has shown to reduce the risk of skeletal-related events in patients with bone metastases from solid tumors. Osteonecrosis of the jaws (ONJ) is an important potential adverse event associated with the use of denosumab or other bone health agents (BHA) like bisphosphonates. In a phase III study conducted to investigate safety and efficacy of Ra223, ONJ was reported in $0.67 \%$ of patients treated with Ra223 and in $0.33 \%$ of patients who received placebo; however, all patients with ONJ were also exposed to prior or concomitant BHA. Furthermore, denosumab has been associated with prolonged survival in patients treated with Ra223, and a BHA therapy should be considered during Ra223 treatment in order to prevent Ra223 induced bone fractures. Considering that background, we report the case of a 67-year-old man with metastatic CRPC already exposed for almost 4 years to denosumab, who developed ONJ during concomitant Ra223 therapy. Despite contrasting findings in real life experiences, our case suggests to carry out a close monitoring of oral health in patients receiving Ra223 with previous long exposure to denosumab.

\section{BACKGROUND}

Radium-223 dichloride (Ra223) is an alpha-emitting radionuclide approved for the treatment of patients with castrationresistant prostate cancer (CRPC) with symptomatic bone metastases. Acting as a calcium mimetic by forming complexes with the bone mineral hydroxyapatite in areas of amplified osteoblastic activity, it directly delivers ionizing radiation to areas of bone metastases. ${ }^{[1]}$

Denosumab is a human monoclonal antibody that targets with high affinity and specificity RANKL, a key mediator of osteoclast formation, function, and survival..[2] By binding RANKL, denosumab inhibits osteoclast activity, resulting in reduced bone resorption and tumor-induced bone destruction. Through these properties, it showed to reduce the risk of skeletal-related events in patients with bone metastases from solid tumors. ${ }^{[3]}$ 
An important potential adverse event associated with the use of denosumab or other bone health agents (BHA) like bisphosphonates (e.g. zoledronate) is Osteonecrosis of the jaws (ONJ), reported in 0.9-10\% patients with cancer receiving denosumab.[4][5]

In the Phase III ALSYMPCA trial, which investigated safety and efficacy of Ra223 in men with metastatic CRPC, ONJ was reported in $0.67 \%$ of patients treated with Ra223 and in $0.33 \%$ of patients who received placebo; however, all patients with ONJ were also exposed to prior concomitant BHA and prior chemotherapy. ${ }^{[6]}$

Furthermore, data showed that metastatic CRPC patients treated with Ra223 with concomitant denosumab had longer overall survival than patients who received Ra223 alone, ${ }^{[7]}$ and a BHA therapy should be considered during Ra223 treatment in order to prevent Ra223 induced bone fractures. ${ }^{[8]}$

Considering that background, we report the case of a metastatic CRPC patient, already exposed for almost 4 years to Denosumab, who developed ONJ during concomitant Ra223 therapy.

\section{CASE REPORT}

A 60 years-old man was diagnosed with prostate cancer in 2010. Staging exams showed the presence of metastases to pelvic bones and inguinal lymph nodes, so in July 2010 the patient started Androgen Deprivation Therapy (ADT). In 2013 the patient underwent an 18F-Choline-PET/CT scan that showed bone disease progression so, from July 2013 to May 2014, the patient received a first-line chemotherapy with docetaxel (50 mg/m2) every two weeks + prednisone (10 mg/day). The treatment was suspended after 16 administrations with good biochemical and radiological response but with severe epiphora and moderate asthenia. From June 2014 the patient continued ADT adding bicalutamide (150 mg/day). 3 months after being subjected to a double tooth extraction and subsequent maxillofacial evaluation, in April 2014 the patient also started treatment with denosumab every 28 days. Due to biochemical progression, from February 2015 to December 2017 the patient underwent second-line therapy with abiraterone acetate $(1000 \mathrm{mg} /$ day $)+$ prednisone $(10 \mathrm{mg} / \mathrm{day})$, which was suspended for clinical and radiological progression of bone disease and, in January 2018, the patient started a new treatment with Ra223 in association to ADT. After 3 months of Ra223 therapy the patient started complaining about jaw pain, so he performed a panoramic RX and a cone-beam CT scan but no pathological findings were observed; after a new maxillofacial evaluation, the patient started antibiotic therapy with a progressive improvement of symptoms. However the patient preventively suspended denosumab, but completed Ra223 therapy in June 2018. At the end of treatment with Ra223 the clinical conditions of the patient worsened, with the development of asthenia and severe anemia, requiring blood transfusions. In October 2018 the patient complained of a recurrence of jaw pain for which he performed a new conebeam CT scan which finally detected the presence of ONJ; at the oral examination the appearance of exposed bone was found, so the patient underwent a new antibiotic therapy. At the same time, he had a disease progression which led to a further aggravation of his condition so he was offered best supportive care; the patient died a few weeks later.

\section{DISCUSSION}

Osteonecrosis of the jaws is a BHA-related adverse event that can occur in up to $7 \%$ of bisphosphonate-treated cancer 
patients and up to $10 \%$ of denosumab-treated cancer patients [5]. Although the exact mechanism of the onset of ONJ is still unknown, several risk factors have been suggested to be associated with the occurrence of ONJ in patients treated with BHA. A history of tooth extraction, poor oral hygiene, and/or use of a dental appliance was linked to the onset of ONJ in patients treated with denosumab or zoledronate. ${ }^{[9]}$ Corticosteroids and chemotherapy have also been implicated in the development of ONJ. Data from three phase-III trials of metastatic cancer patients treated with denosumab or zoledronate showed there was some association of ONJ with the use of corticosteroids, while no association with the use of chemotherapy has been described. ${ }^{[10]}$ Otherwise, in a retrospective analysis of bone metastatic prostate cancer patients treated with zoledronate, chemotherapy with docetaxel was also associated with a strong, but not statistically significant, trend towards increased risk of ONJ. ${ }^{[11]}$ In the same study, the length of exposure to zoledronate ( $\geq 9$ administrations) was associated with an increased frequency of ONJ. Incidence rate of ONJ has also been associated to the duration of exposure to denosumab in patients with prostate cancer. ${ }^{[12][13]}$

Regarding the occurrence of ONJ during Ra223 therapy, as mentioned above, it was doubled in patients who received Ra223 compared to placebo, although it was a rare event and possibly related to prior o concomitant $\mathrm{BHA}$ and prior chemotherapy [6]. Moreover, a possible synergism between ra223 and bisphosphonates has also been suggested and the concurrent use of zoledronate and Ra223 is permitted. ${ }^{[14]}$

Nevertheless, no consensus exists regarding the use of Ra223 in patients with ONJ induced by BHA, and the few clinical experiences reported are mainly limited to the use of bisphosphonates. Herlofson et al. reported the case of metastatic CRPC patient who had a severe deterioration of bisphosphonate-related ONJ after Ra223 was started, suggesting a possible cumulative effect between Ra223 and bisphosphonate therapy. ${ }^{[15]}$ Otherwise Dionisi et al., reporting 2 cases of patients with zoledronate-induced ONJ treated with Ra223 for metastatic CRPC-related bone metastases, suggested that there are no significant contraindications to the use of Ra223 in patients with ONJ, with a little worsening of osteonecrosis which is negligible compared with the benefits induced by Ra223. ${ }^{[16]}$ Moreover, Costa et al reported the case of a 54-yearold woman with breast cancer with bone metastases and zoledronate-induced ONJ, who experienced a self-extraction of necrotic bone during treatment with Ra223 in absence of pain or suppuration, suggesting Ra223 may have an adjuvant role in the treatment of cancer patients with ONJ. ${ }^{[17]}$

It is clear that the patient described in our report had several risk factors associated with ONJ: the history of tooth extraction, the previous treatment with corticosteroids and taxane-based chemotherapy, and the long-lasting exposure to denosumab could all have contributed to the onset of ONJ, which then occurred during Ra223 therapy. For these reasons, we are not able to establish a causal relationship between the occurrence of ONJ and therapy with Ra223, albeit there was a temporal consequence. Moreover we cannot exclude that Ra223 may have triggered the onset of osteonecrosis on a condition already deteriorated by other factors, although the exact mechanism would be unknown.

\section{CONCLUSIONS}

Given the undoubted benefit that Ra223 therapy can bring in CRPC with bone metastases and for which it should always be considered, and taking into account the contradictory evidences regarding the use of Ra223 in patients with BHArelated ONJ, our experience suggests to carry out a close monitoring of oral health in patients receiving Ra223 with 
previous long exposure to denosumab.

\section{References}

1. 'Sten Nilsson, Roy H. Larsen, Sophie D. Fosså, Lise Balteskard, et al. (2005). First Clinical Experience with $\alpha$-Emitting Radium-223 in the Treatment of Skeletal Metastases. Clin Cancer Res, vol. 11 (12), 4451-4459. doi:10.1158/10780432.ccr-04-2244.

2. ^D.L Lacey, E Timms, H.-L Tan, M.J Kelley, et al. (1998). Osteoprotegerin Ligand Is a Cytokine that Regulates Osteoclast Differentiation and Activation. Cell, vol. 93 (2), 165-176. doi:10.1016/s0092-8674(00)81569-x.

3. 'Alison T. Stopeck, Allan Lipton, Jean-Jacques Body, Guenther G. Steger, et al. (2010). Denosumab Compared With Zoledronic Acid for the Treatment of Bone Metastases in Patients With Advanced Breast Cancer: A Randomized, Double-Blind Study. JCO, vol. 28 (35), 5132-5139. doi:10.1200/jco.2010.29.7101.

4. `Ana Boquete-Castro, Gerardo Gómez-Moreno, José Luis Calvo-Guirado, Antonio Aguilar-Salvatierra, et al. (2015). Denosumab and osteonecrosis of the jaw. A systematic analysis of events reported in clinical trials. Clin. Oral Impl. Res., vol. 27 (3), 367-375. doi:10.1111/clr.12556.

5. 'Tine Loyson, Thomas Van Cann, Patrick Schöffski, Paul M. Clement, et al. (2017). Incidence of osteonecrosis of the jaw in patients with bone metastases treated sequentially with bisphosphonates and denosumab. Acta Clinica Belgica, vol. 73 (2), 100-109. doi:10.1080/17843286.2017.1348001.

6. ^Case Medical Research. (2020). Human medicines European public assessment report (EPAR): Xofigo, radium Ra223 dichloride, Prostatic Neoplasms, Date of authorisation: 13/11/2013, Revision: 10, Status: Authorised. CMR. doi:10.31525/cmr-267ec37.

7. ^Fred Saad, Joan Carles, Silke Gillessen, Axel Heidenreich, et al. (2016). Radium-223 and concomitant therapies in patients with metastatic castration-resistant prostate cancer: an international, early access, open-label, single-arm phase 3b trial. The Lancet Oncology, vol. 17 (9), 1306-1316. doi:10.1016/s1470-2045(16)30173-5.

8. ^https://www.ema.europa.eu/en/documents/product-information/xofigo-epar-product-information_it.pdf.

9. 'Alison T. Stopeck, Karim Fizazi, Jean-Jacques Body, Janet E. Brown, et al. (2015). Safety of long-term denosumab therapy: results from the open label extension phase of two phase 3 studies in patients with metastatic breast and prostate cancer. Support Care Cancer, vol. 24 (1), 447-455. doi:10.1007/s00520-015-2904-5.

10. ^ F. Saad, J.E. Brown, C. Van Poznak, T. Ibrahim, et al. (2012). Incidence, risk factors, and outcomes of osteonecrosis of the jaw: integrated analysis from three blinded active-controlled phase III trials in cancer patients with bone metastases. Annals of Oncology, vol. 23 (5), 1341-1347. doi:10.1093/annonc/mdr435.

11. 'Cinzia Ortega, Filippo Montemurro, Roberto Faggiuolo, Roberto Vormola, et al. (2007). Osteonecrosis of the jaw in prostate cancer patients with bone metastases treated with zoledronate: A retrospective analysis. Acta Oncologica, vol. 46 (5), 664-668. doi:10.1080/02841860601185917.

12. 'Matthew R Smith, Fred Saad, Robert Coleman, Neal Shore, et al. (2012). Denosumab and bone-metastasis-free survival in men with castration-resistant prostate cancer: results of a phase 3, randomised, placebo-controlled trial. The Lancet, vol. 379 (9810), 39-46. doi:10.1016/s0140-6736(11)61226-9.

13. 'Matthew R. Smith, Fred Saad, Stephane Oudard, Neal Shore, et al. (2013). Denosumab and Bone Metastasis-Free 
Survival in Men With Nonmetastatic Castration-Resistant Prostate Cancer: Exploratory Analyses by Baseline ProstateSpecific Antigen Doubling Time. JCO, vol. 31 (30), 3800-3806. doi:10.1200/jco.2012.44.6716.

14. ' , Joelle El-Amm. (2016). Targeting Bone Metastases in Metastatic Castration-Resistant Prostate Cancer. CMO. doi:10.4137/cmo.s30751.

15. ^Bente Brokstad Herlofson, Gry Karina Kjølle, Kristine Løken Westgaard, Ayca M. Løndalen, et al. (2018). Osteonecrosis of the Jaw in a Patient With Bone Metastatic Prostate Cancer After Long-term Bisphosphonate Treatment With Severe Deterioration Following Radium-223. Clinical Genitourinary Cancer, vol. 16 (5), 328-331. doi:10.1016/j.clgc.2018.05.003.

16. 'Valeria Dionisi, Chiara Bellarosa, Raffaele Cardano, Elisa Lodi Rizzini, et al. (2019). Use of Radium-223 Dichloride in Patients With Osteonecrosis of the Jaw Induced by Zoledronic Acid: Report of 2 Cases. Clinical Genitourinary Cancer, vol. 17 (3), e612-e617. doi:10.1016/j.clgc.2019.03.002.

17. ^RenatoPatrizio Costa, Vincenzo Tripoli, Alessandro Princiotta, Alessandra Murabito, et al. (2019). Can radium 223 be a conservative non-surgical management of medication-related osteonecrosis of the jaw?. World J Nucl Med, vol. 18 (3), 307. doi:10.4103/wjnm.wjnm_7_19. 